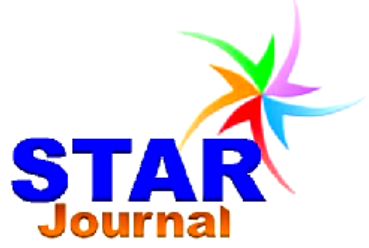

\author{
ISSN: 2226-7522(Print) and 2305-3327 (Online) \\ Science, Technology and Arts Research Journal \\ Jan-March 2012, 1(1): 18-25 \\ www.starjournal.org \\ Copyright $\odot 2012$ STAR. All Rights Reserved
}

Original Research

\title{
Preliminary Study on the Epidemiology of Tuberculosis in Nekemte and Its Surroundings -Western Ethiopia
}

\author{
Eyasu Ejeta $^{1,2^{*}}$, Mengistu Legesse ${ }^{1}$, Gobena Ameni ${ }^{1}$ \\ ${ }^{1}$ Aklilu Lemma Institute of Pathobiology, Addis Ababa University, Addis Ababa, Ethiopia \\ ${ }^{2}$ Faculty of Medical Sciences, Wollega University, Post Box No: 395, Nekemte, Ethiopia
}

\begin{tabular}{|c|c|}
\hline Abstract & Article Information \\
\hline $\begin{array}{l}\text { Epidemiological study for Tuberculosis (TB) has been conducted in developed and } \\
\text { developing countries to accesses the burden of the disease in their countries. } \\
\text { However, this study was lacking in Nekemte and its surroundings, western Ethiopia. } \\
\text { Therefore, present study was designed for cross-sectional study to generate } \\
\text { preliminary epidemiological information on TB in Nekemte and its surroundings, } \\
\text { Western Ethiopia. Clinical examination, chest } X \text {-ray, acid-fast staining, culture and } \\
\text { polymerase chain reaction (PCR) were used in the study. In addition, five years } \\
\text { Nekemte Hospital and Nekemte Health center data were analyzed to assess the five } \\
\text { years situation of TB, TB and HIV co-infection, and treatment outcome. The results of } \\
\text { the study reveals that out of } 30,945 \text { TB cases registered from } 2003-2008 \text {, } 19,494 \\
\text { ( } 63 \% \text { ) had pulmonary TB (PTB) and } 11,451 \text { ( } 37 \% \text { ) suffered from extra-pulmonary TB } \\
\left.\text { (EPTB) ( } x^{2}=81.936 ; p<0.001\right) \text {. From } 19,494 \text { registered PTB cases, smear positive PTB } \\
\text { accounted for } 2,462(12.6 \%) \text { of cases. The incidences of all forms of TB were } \\
\text { gradually declining in the last five years, while the trend of smear positive TB was } \\
\text { almost constant, according to retrospective analysis. The disease was also showed to } \\
\left.\text { be more prevalent in } 15-64 \text { age groups ( }{ }^{2}=288.035 ; p<0.001\right) \text {. In addition, around } \\
11.5 \% \text { of HIV positive patients were positive for TB. During two months study period, } \\
520 \text { new TB cases were registered. Out of these, } 68 \text { (13.1\%) were smearing positive. } \\
\text { The extrapolated annual incidence of clinically diagnosed and smear positive TB in the } \\
\text { study area were } 124.8 / 100,000 \text { and } 6.32 / 100,000 \text { population, respectively. Out of } 25 \\
\text { isolate analyses by PCR, } 15 \text { were } M \text {. tuberculosis (Mtb), } 4 \text { were mixed with Mtb and } \\
M \text {. bovis (Mb), } 2 \text { were unknown band and five were negative. The retrospective and } \\
\text { present study showed that the significance of TB problem in the study area was not at } \\
\text { the level to be undermined }\end{array}$ & $\begin{array}{l}\text { Keywords: } \\
\text { Tuberculosis } \\
\text { Nekemte } \\
\text { AFB } \\
\text { PCR } \\
\text { Culture } \\
\end{array}$ \\
\hline
\end{tabular}

\section{INTRODUCTION}

Tuberculosis (TB), one of the most wide spread infectious disease, is the leading cause of death in the world. It has been known in human history since ancient time (Zink et al., 2003) and still it remains an important public health problem (WHO, 2008). According to the World Health Organization (WHO), currently, nearly 2 billion peoples (approximately one-third of the world's population) have been exposed to TB pathogen (WHO, 2008). Annually, 9.2 million people become ill with TB, and 1.7 million people die from the disease worldwide (CDC, 2005; WHO, 2008). Developing countries bear the highest burden of the TB epidemic. An estimation of $95 \%$ of TB cases and $98 \%$ of TB deaths occurs in the developing parts of world, where peoples are especially vulnerable to TB because of poor living conditions and limited access to treatment (WHO, 2006; Raviglione and O'Brien, 2004). Estimation shows that in sub-Sahara Africa alone, there are 1.3 million new cases of TB and 50,000 deaths annually (Porter and Adam, 1992). The young and most 
Eyasu Ejeta et al.,

productive segments of the population is seriously affected by the disease with the infection rate goes up to $50 \%$ (Styblo et al., 1996).

In Ethiopia, TB is among the leading cause of mortality and morbidity (Azbite, 1991; FMOH, 2008). Over one third of the population has been exposed to TB (WHO, 2005), and it has becoming a disease of major public health important, due to low health services coverage and poorly developed health information system in the country (WHO, 2005). Sero-prevalence rates of HIV among adult TB patients are $11 \%$ and $31 \%$ according to $\mathrm{WHO}$ report and more recent nation data from 1999 E.C., respectively (FMOH, 2008).

Nekemte Hospital is a Referral hospital which is found in Nekemte Referral Town, East Wollega Zone, Western Ethiopia. It was estimated that over 2.5 million of people underwent treatment in this hospital. Currently, the Hospital gives diagnostic services for TB patients and it has not yet started the direct observed treatment short course (DOTS) program. Even though, there were no documents so far that shows specific figure of the TB problem in these part of Ethiopia, the hospital and other health center data show that the disease is a leading cause of mortality and morbidity. Similarly, the HIVIAIDS seroprevalence for this area was found to be high according to surveillance conducted in the past which may be one of a contributing factor for this high public important of TB in the district. This study was, therefore, designed to generate preliminary epidemiological information on TB in Nekemte and its surroundings -Western Ethiopia.

\section{MATERIALS AND METHODS}

\section{Study Area and Study Participants}

This cross-sectional study was conducted at Nekemte Referral Hospital, Western Ethiopia. The study participants were patients exhibiting the typical clinical signs and symptoms of TB visiting the out patients department (OPD) of the Hospital that with those have no history of starting anti-TB, able to expectorate sputum, above the age of five years, and voluntary to participate in the study.

\section{Sample Size}

Sample size was determined based on the period of data collection (two months). During
Sci. Technol. Arts Res. J., Jan-March 2012; 1(1): 18-25

these periods, 520 clinical suspected TB cases were visited the hospital and investigated. The sputum samples were collected and cultured for 153 TB suspected patients because of limitation of resource to culture samples from all study patients.

\section{Diagnosis and Laboratory Procedure}

Clinical examination, chest radiography and microscopic examination of the study patient conducted at Nekemte Hospital as routine diagnostic procedure, and those patients positive for the disease was order to inter TB drug regime based on the discussion of physician.

For isolate and characterize species of mycobacterium from PTB at the study area, the collected sputum sample was stored at deep freezer $\left(-20^{\circ} \mathrm{C}\right)$ immediately until transported to Aklilu Lemma Institute of Pathobiology, Addis Ababa University for TB culture after acid fast examination done at the study site. The stored sputum samples were transported using cold chains every seven days to the TB laboratory for culture and $P C R$ processing.

Lowenstein-Jensen (LJ) media was used for culturing the sputum samples. The sputum sample was decontaminated by adding equal volume of $4 \% \mathrm{NaOH}$ solution, mixed very well and centrifuged at $3000 \mathrm{rpm}$ for $10 \mathrm{~min}$. The supernatant was decanted and the sediment was resuspended by mixing. Then a drop of phenol red was added to the suspension as indicator, and neutralized by $2 \mu \mathrm{HCL}$ through adding drop by drop until the color of the phenol red changed from purple to yellow. Then, one to two drops of the inoculums was added to the LJ medium. The inoculated tube was incubated in slant position with the tube screw caps closed for one week at $37{ }^{\circ} \mathrm{C}$, and then put in up-right position until complete growth is seen in the some condition.

For isolate and characterize species of mycobacterium from PTB, RD4 deletion typing was used based on method described by Cleaveland et al. (2007). The primers used in this study was RD4 flank FCTCGT CGAAGGCCACTAAAG; RD4 flan RAAGGC GAACAGATTCAGCAT; and RD4 intF; ACA GGCTGGCGAAGTATAGC. If RD4 is present (i.e $M$. tuberculosis and $M$. africanum) a productive size of 335bp (RD4-intF + RD4flankR) was obtained; if it is deleted ( $M$. bovis); then a product of 446bp (RD4-flankF + RD4flankR) was obtained. 
Eyasu Ejeta et al.,

\section{Retrospective Data Record Analysis}

Nekemte Hospital case book was used to assess the situation of TB in the last five years (2003-2008) in Nekemte and its surroundings. The forms of TB, sex and age pattern, as well as TB and HIV co-infection were assessed. Regarding treatment outcome of TB patients in Nekemte and its surrounding for the last five years (2003-2008), data were collected from Nekemte Health Center since the Nekemte Hospital was not yet started the DOTS programs and the data were not available.

\section{Ethical Consideration}

The study was ethically cleared by the Institutional Ethical Clearance Committee, Aklilu Lemma Institute of Pathobiology, Addis Ababa University. After the detailed purpose of the study was explained to each presumptive patient and those voluntary to participate in the study are confirmed their willingness by giving their oral or written consents.

\section{Statistical Analysis}

All data were analyzed by using SPSS for windows version 15.0 and Microsoft Excel. Descriptive statistics was used for analysis and expression of the result. Chi-square tests were used to compare difference between groups, and comparisons (difference) at $p<0.05$ were considered as statistically significant in this study. Strength of agreement between the TB diagnoses methods was assessed by using kappa.

\section{RESULTS}

\section{Result of Retrospective Data Analysis \\ Demographic Data}

Demographic data of TB patients were presented in Figure 1. Out of 30,945 TB cases registered in the last five years, 16,306 $(52.7 \%)$ were males $\left(X^{2}=30.205 ; p<0.001\right)$, while out of all PTB and EPTB registered, $55.6 \%$ were females and $52 \%$ were males, respectively. The annual trends of TB for both sexes were gradually declined in the last five years.

The mean age of all registered TB case was 35.35. In both form of TB (ETB and EPTB) the age group that most common affected by this disease was $15-44(47.10 \%)$ followed with $45-$ $64(35.50 \%)$, and $5-15(14.39 \%)$. But the
Sci. Technol. Arts Res. J., Jan-March 2012; 1(1): 18-25

disease was less commonly in the age groups less than five years $(0.74 \%)$ and greater than sixty four years age $(2.72 \%)$ groups $\left(x^{2}=\right.$ 288.035; $p<0.001$ ) (Figure 2).

In both sexes the disease was showed to be higher in 15-44 and followed with 45-64 ages group. However, in below 15 age groups, around $54.4 \%$ active TB recorded in female while in above 15 years old TB was prevalent in males $\left(X^{2}=30.205 ; p<0.001\right)$.

\section{Pattern of Tuberculosis in Nekemte and its Surroundings}

Out of 30,945 TB cases that registered from 2003-2008, 19,494 (63\%) had PTB and 11,451 $(37 \%)$ suffered from EPTB $\left(X^{2}=81.936 ; p<0.001\right)$. From 19,494 PTB cases in the last five years, smear positive PTB accounted for $12.6 \%$. The trend of TB incidence in the last five years showed gradual decline per year with slight variation between years (Figure 3). As indicated by Figure 3 , the incidence of PTB was higher $(63 \%)$ than that of EPTB $\left(X^{2}=81.936 ; p<0.001\right)$. The trend of smear positive PTB was found to be almost constant in the last five years while smear negative PTB showed gradually declining $\left(x^{2}=81.936 ; p<0.001\right)$.

Trend of TB Treatment Outcomes in Nekemte and its Surrounding

The trends of relapse, death and defaulter of TB chemotherapy in Nekemte and its surroundings were shown by the Figure 4 . The numbers of relapse and death cases were generally showed increment in the last five years. Unlikely to the number of relapse and death cases, the defaulter cases showed gradual declining in the last five years $\left(x^{2}=108.76, p<0.001\right)$. Regarding to failure cases in the study area, there was no any cases that recorded as treatment failure.

Even though, the overall sex difference in treatment outcome was not showed any statistical significant difference between both sexes $\left(x^{2}=2.86 ; p>0.05\right)$, the relapse cases were slightly higher in females $16(53.3 \%)$ than in males, while defaulter $46(52.3 \%)$ and death $44(58.7 \%)$ cases were occurred highly in males. 


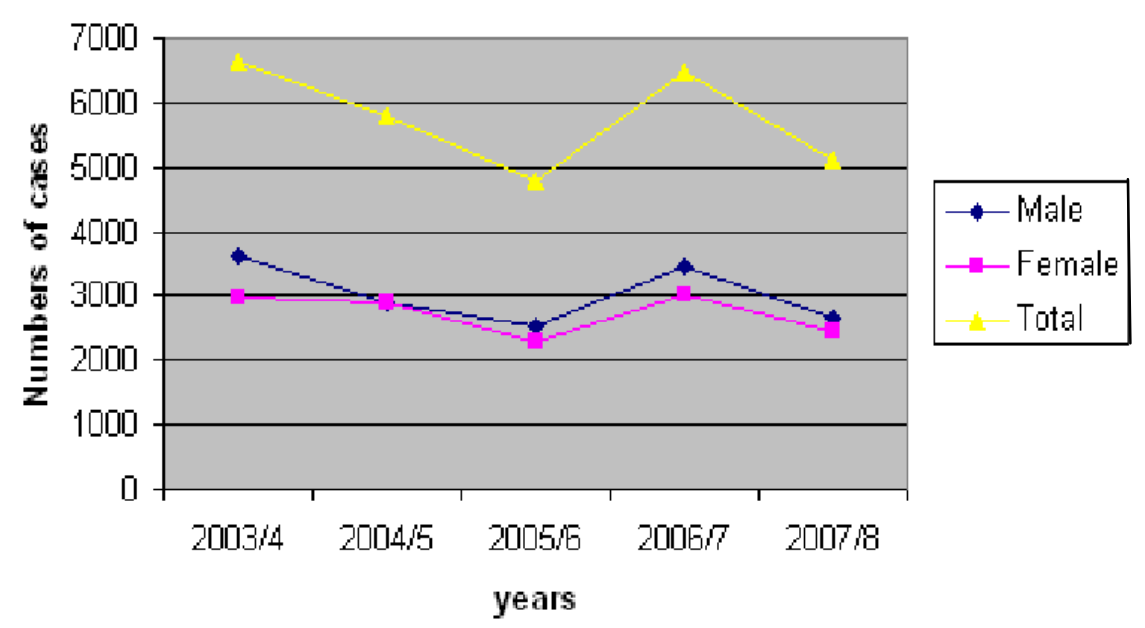

Figure 1. Sex distribution of TB during 2003-08 in Nekemte and its surroundings.

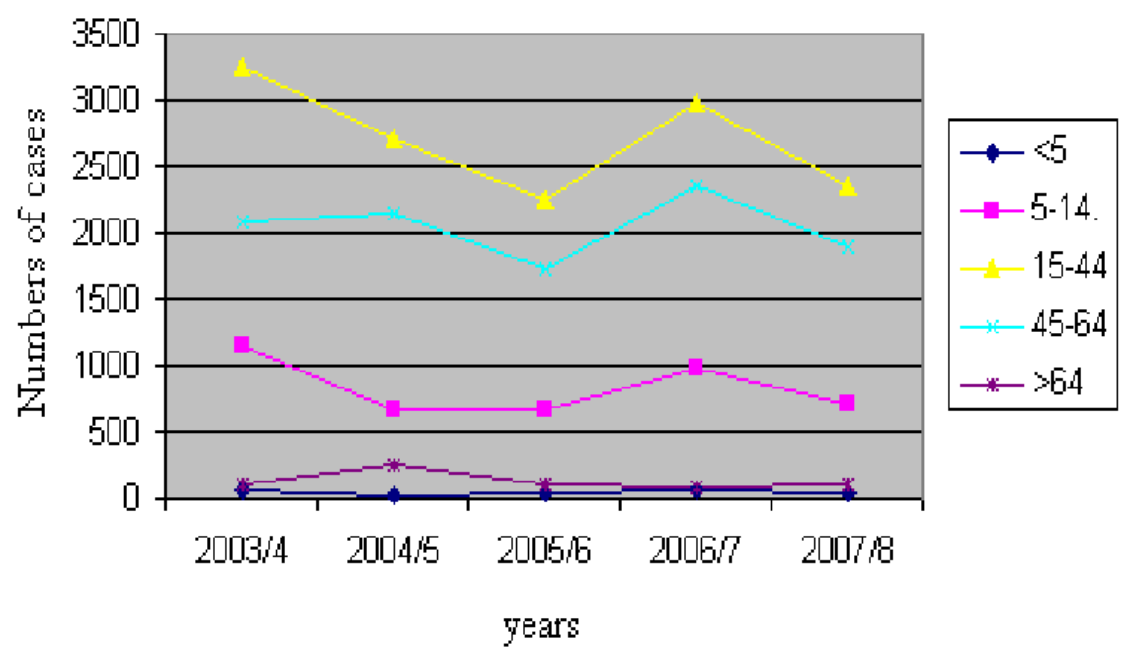

Figure 2. Age distribution of TB cases that registered during 2003-08 in Nekemte \& its surroundings.

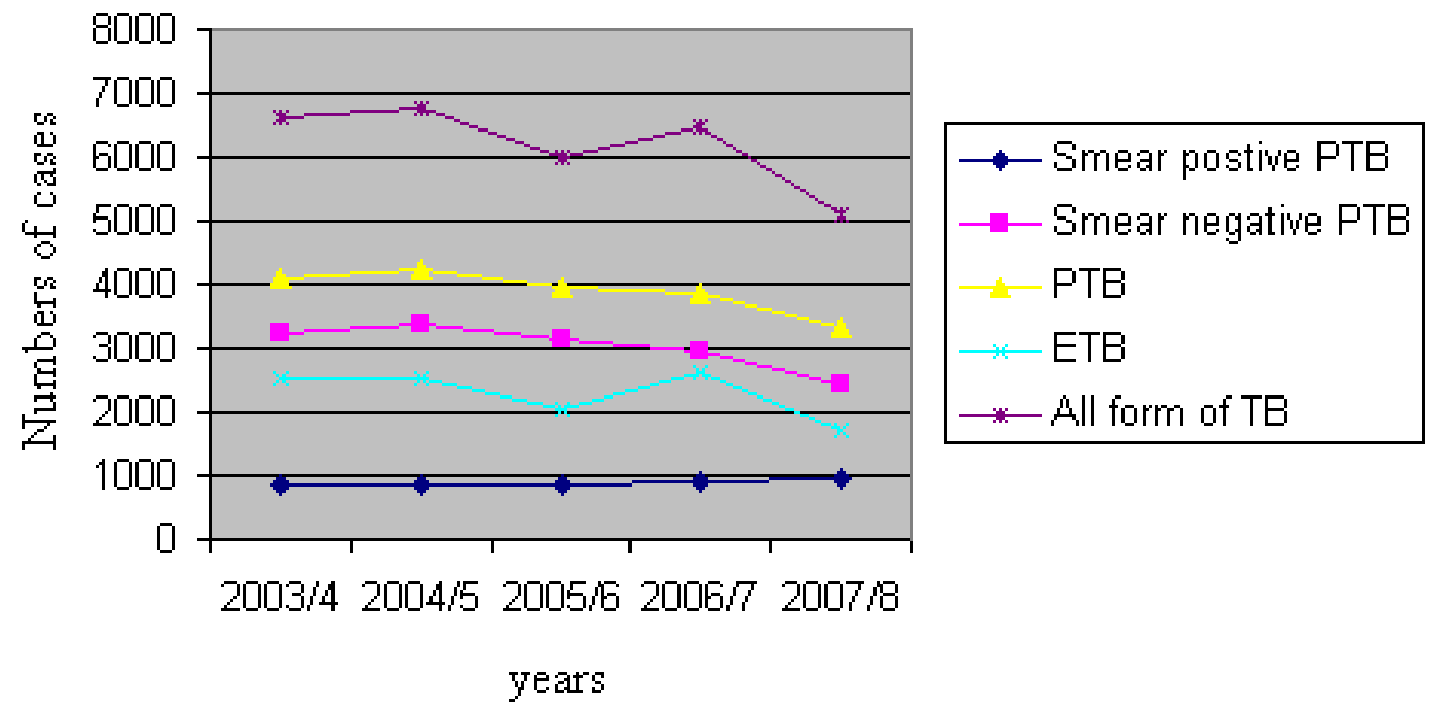

Figure 3. Trends of all form of TB, PTB, EPTB, smear positive and smear negative PTB that registered in the last five year in Nekemte and its surroundings. 


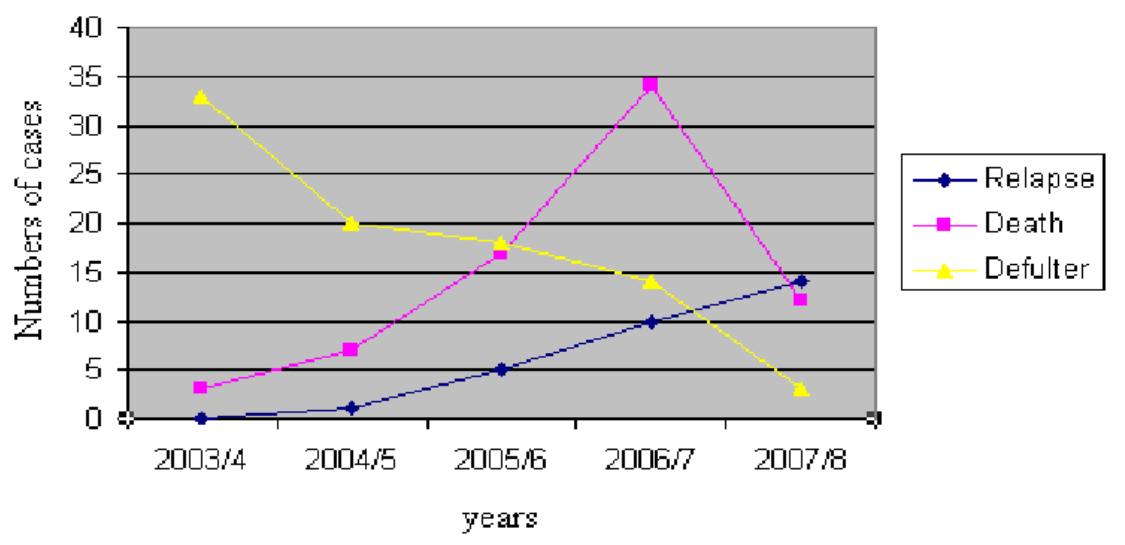

Figure 4. Trends of relapse, death and defaulter cases for TB treatment in Nekemte and its surroundings during 2003-08.

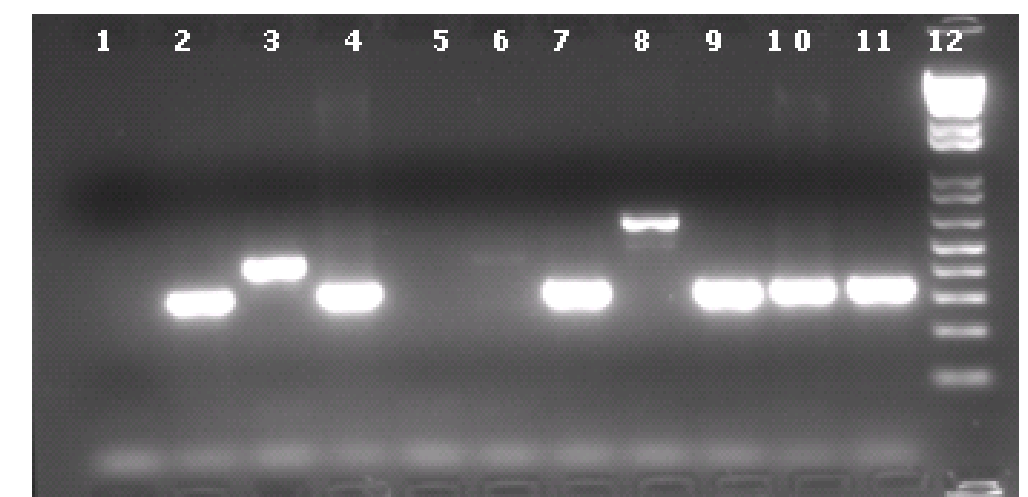

Line 1= non template control, Line 2= Mtb positive control, Line 3 = Mb positvecontrol, Line 4-11 =sample, Line12 =1 kb Ladder

Figure 5. RD4 gel results of some representative samples.

\section{Prevalence of TB-HIV Co-infection in Nekemte and its Surroundings}

The data for TB and HIV co-infection were only available from September 2007 to October 2008 at Nekemte Hospital. During this period, 1302 HIV positive patients were tested for TB and out of these $146(11.21 \%)$ were found to be positive for TB as indicated in Table 2, Therefore, the prevalence of TB and HIV coinfection during this period of time in Nekemte and its surroundings was found to be $11.21 \%$ (146/1302).

\section{Incidence of Tuberculosis in Nekemte and its Surroundings}

On the base of clinical examination, 520 new TB cases were recorded during the two months of data collection period. Out of these, 68 $(13.1 \%)$ were positive for acid fast staining, and $53 \%$ were males. The mean age of the study subjects were 32.5 and the disease was mostly recorded in 15-44 age groups.
Extrapolation from two month record of TB patients, on average 3,120 new clinically diagnosed and 408 smear positive patients would visit the Nekemte Hospital annually. Thus, the annual incidence of clinically diagnosed and smear positive TB in Nekemte and its surrounding was 124.8/ 100,000 and $16.32 / 100,000$ population, respectively.

\section{Polymerase Chain Reaction (PCR)}

Twenty five (25) isolate were processed for identification by PCR (Figure 5), Out of these, $14(56 \%), 4(16 \%)$ and $2(8 \%)$ were identified to be Mtb, mixed infection of two species (Mtb and $\mathrm{Mb})$ and unknown band, respectively. The remaining five samples $(20 \%)$ were negative by PCR. 


\section{DISCUSSION}

The retrospective data analysis showed that out of all forms of TB registered in the last five years, $53 \%$ were males which was consistent with the report of other studies conducted in different part of the country (Kassu et al., 2007; Shargie and Lindtjorn, 2005) and the recent report of WHO (2008). This result reflects sex difference in the disease risk or access to treatment. The sex distribution of the forms of TB recorded in the present study is similar with that reported in Nepal (Chandrasekhar et al., 2008).

Even though, the mean age of all registered TB cases was 35.35 , in both forms of TB, the age group that most commonly affected by this disease was 15-44 followed with 45-64. But the disease was less commonly registered in the others age groups. This result is consistent with the report of the other studies conducted in southern Ethiopia (Shargie and Lindtjorn, 2005), in Nepal (Chandrashekhar et al., 2008) and from the recent national report $(\mathrm{FMOH}, 2008)$. TB is prevalent in the age group 15-44 as this age group is sexually active may be co-infected with HIV, which is a risk factor for TB. Besides, TB is prevalent in male in all age group except in those years age below 15, which is consistent with the globally age pattern of TB where the number of male TB cases exceed that of female in all age groups except in children (WHO, 2008).

The finding from retrospective study indicated that the overall annual trends of all forms TB in the last five years (2003-2008) were observed to fall gradually. This is similar to the national and global TB incidence according to the recent report of WHO (2008). This could be due to the effect of DOTS program. This study also showed the higher incidence of PTB than the incidence of EPTB. This result supports the study that was conducted in south region Ethiopia where PTB accounted $67 \%$ of all from TB in the area (Shargie and Lindtjorn, 2005), and in northwestern Ethiopia where PTB accounted $64.2 \%$ from all form of TB, regardless of HIV status (Kassu et al., 2007). This result further confirms the presence of similarity in the forms of TB in different part of the country. Contrary to the trend of smear negative PTB, the trend of smear positive PTB was almost constant in the last five year. These could be due to the increasing quality of laboratory services and dissemination of HIVIAIDS in the study area.
Presently, DOTS program was implemented in the study area but the relapse and death cases were generally increasing while the defaulters were gradually declining per years in responding to DOTS program, which was similar to other studies done in south Ethiopia (Shargie and Lindtjorn, 2005), in northwestern shewa (Seyoum, 2007) and in Jijiga district (Mohammed, 2007). Thus, this increasing in the incidence of relapse from these studies in different part of the country may be an indication for dissemination of the drug resistance strain of TB in the country as the national incidence MDR-TB in relapse cases was $12 \%$ (WHO, 2008). The other possible reason could be a rapid dissemination of HIVIAIDS in the area would increase the incidence of relapse and death cases while the implementation of DOTS, increasing of the community knowledge about TB and health facilities in area might have contributed for this change in the defaulter rate.

Even though, overall sex difference in treatment outcome was not statically significant, there were low defaulter cases in females supporting the result of other studies (Shargie and Lindtjorn, 2005; Wally et al., 2001). The presence of slightly higher relapse rate in females and higher death rate in males were also found in this study of TB (Holmes et al., 1998; Hudelson, 1996).

The incidence of TB in HIV positive individuals was found to be $11.21 \%$ in this study, which is higher than the national incidence $(6.3 \%)$, according to the recent WHO report (WHO, 2008). This high incidence of TB and HIV co-infection in Nekemte and its surroundings may be due to the high HIVIAIDS dissemination in the study area that would have the effect on TB progression.

The extrapolated annual incidence of all from and smear positive TB in this study are lower than the national incidence of all form of TB per year $(379 / 100,000$ population) and smear positive TB $(168 / 100,000$ population) as the recent report of WHO (WHO, 2008). This may be due to the extrapolation may not indicate the real picture of TB in the study area as the incidence of TB is not evenly distributed with in 12 month of a year. The other possible reason is the incidence of TB in the study area may be lower than the national TB incidence. 


\section{Eyasu Ejeta et al.,}

PCR result showed that $56 \%$ infections were caused by Mtb while $16 \%$ were caused by mixed infection with Mtb and Mb. This finding showed lower proportion of Mtb infection as compared to other studies conducted in Addis Ababa with $98.37 \%$ (Bruchfeld et al., 2002) and in Nigeria around $85 \%$ (Cadmus et al., 2006) TB infection was caused by Mtb.

\section{CONCLUSION}

Result of the retrospective study showed the significance of the disease in the study area. The trends of all forms of TB, and defaulters were gradually declining in the last five years, which may be a promising achievement in the control of the disease in this area. However, the disease was more commonly in the productive age (15-64) groups of the population thereby affecting the economic development. In addition, the detection rate (incidence of smear positive TB cases) was almost constant while relapse and death rate were rising in associated to dissemination of HIV in the area. Thus, farther detail study has to be done in future to identify the contributing factors and the strain of the mycobacterium that circulate in the study area.

\section{Acknowledgements}

We would like to thank Dr. Teshome Abayne head of Nekemte Hospital and Nekemte Hospital laboratory staff members for their unreserved assistance during collection of data and specimen and to Hilu Getu, Tedesse Regassa and Bezabih Fetene for their valuable technical support during culturing the samples.

\section{REFERENCES}

Azbite, M. (1991). Tuberculosis survey in Ethiopia. Akaka. 67: 8539-8544.

Bruchfeld, J., Aderaye, G., Palme, I. B.,Bjorvatn,B., Ghebremichael, S., Hoffner, S., Lindquist, L. (2002). Molecular Epidemiology and Drug Resistance of Mycobacterium tuberculosis Isolates from Ethiopian Pulmonary Tuberculosis Patients with and without Human Immunodeficiency Virus Infection. Journal of clinical microbiology 40: 16361643.

Cadmus, S., palmer, S., Okker, M., Dale, J., Gover, K., Smith, N., Jahans, K., Glyn, H., V.Gordon, S. (2006). Molecular analysis of human and bovine tuberculosis bacilli from a local setting in Nigeria. Journal of clinical microbiology 44: 29-34.

Centers for Disease Control (CDC). (2005). Fact Sheet: Tuberculosis in the United States.
Sci. Technol. Arts Res. J., Jan-March 2012; 1(1): 18-25

Chandrashekhar, T. S., Kishore, V. P., Sharat, C. V., Hari, S. Joshi., Michael, N. B. (2008). Comparison of pulmonary and extra-pulmonary tuberculosis in Nepal-a hospital-based retrospective study. BioMed Center Infectious Disease 8: 8-25.

Cleaverland, S., Shaw, D. J., Mfinanga, S. g., Shirima, G., Kazawala, R. R., Eblate, E., Sharp, M. (2007). Mycobactrium bovis in the rural Tanzania: Risk factors for infections in human and cattle population. Tuberculoses 86: 30-46.

Fedral Minster of Health (FMOH). (2008). Manual of Tuberculosis, Leprosy and TB/HIV prevention and control programme. $4^{\text {th }}$ edition. Pp1-90.

Holmes, C. B., Hauster, H., Nunn, P. (1998). A review of sex difference in the epidemiology of tuberculosis. Intentional Journal of Tuberculosis and Lung Disease 2: 96-104.

Hudelson, P. (1996). Gender differences in tuberculosis: the role of socio-economic and cultural factors. Tuberculosis and Lung Disease 77: 391-400.

Kassu, A., Mengistu, G., Ayele, B., Diro, E., Mekonnen, F., Ketema, D., Moges, F., Mesfine, T., Getachew, A., Ergicho, B., Elis, D., Aseffa, A, Wondmikun, Y., Ota, F. (2007). Co-infection and clinical manifestation of tuberculosis in human immunodeficiency virus -infected and -uninfected adults at $\mathrm{s}$ teaching hospital, North West Ethiopia. Journal of Microbiology, immunology and Infectious 40: 116-122.

Mohammed, I. (2007). Preliminary study on tuberculosis in Jijiga district, Eastern Ethiopia, MSc thesis, Aklilue Lemma Institute of Path biology, Addis Ababa University, Ethiopia.

Porter, J., Adam, K. (1992). Tuberculosis in Africa in the ADIS era: the role of chemotherapyaxis. Journal of Transaction of the Royal Society of Tropical Medicine and Hygiene 86: 466-469.

Raviglione, M.C., O'Brien, R.J. (2004). Tuberculosis, In: Kasper, D. L., Braunwald, E., Fauci, A. S., Hauser, S. L., Longo, D. L., Jameson, J. L., Isselbacher, K. J. (Eds.). Harrison's Principles of Internal Medicine, $16^{\text {th }}$ Edition, McGraw-Hill Professional. Pp 953-966.

Seyoum, T. S. (2007). Study on tuberculosis in North Western Shewa, Central Ethiopia, M.Sc thesis, Aklilue Lemma Institute of Path biology, Addis Ababa University, Ethiopia.

Shargie, B. E., Lindtjorn, B. (2005). DOTS improves treatement out comes and servies coverage for tuberculosis in Ethiopia: a retrospective tend analysis. BioMed Center Public Health 5: 62-73.

Styblo, K., Frencly, D., Petty, T. (1996). Tuberculosis control and surveillance. In: Recent advance in the respiratory medicine. Edinbur Churchill living stone 5: 77-108. 
Eyasu Ejeta et alo,

Wally, J. D., Khan, M. A., Newell, J. N., Khan, M. H. (2001). Effect of direct observed treatment component of DOTS for tuberculosis: a randomized controlled trail in Pakistan. Lancet 357: 664-669.

World Health Organization (WHO). (2005). Global tuberculosis control: surveillance, planning, financing. World Health Organization report, Geneva: (WHO/HTM/TB/2005.349).

World Health Organization (WHO). (2008). Global tuberculosis control: surveillance, planning, financing: WHO report 2008: (WHO/HTM/TB/2008.393).
Sci. Technol. Arts Res. J., Jan-March 2012; 1(1): 18-25

World Health Organization (WHO). (2006). Global tuberculosis control-surveillance, planning, financing: WHO report.

Zar, H. J. ( 2004). Tuberculosis in developing world. Pediatric Pulmonary Supplement 26: 53-54.

Zink, A., Sola, C., Resichl, U., Grabner, W., Rastogi, N., Wolf, H., Nerlich, A. (2003). Characterization of Mycobacterium tuberculosis complex DNAs from Egyptian mummies by spoligotypin. Journal of Clinical Microbiology 41: 359-36. 